\title{
TOTAL COLORINGS OF EMBEDDED GRAPHS WITH NO 3-CYCLES ADJACENT TO 4-CYCLES
}

\author{
BING WANG \\ Department of Mathematics \\ Zaozhuang University, Shandong, 277160, China \\ e-mail: hellobingzi@163.com. \\ JIAN-LIANG WU \\ School of Mathematics \\ Shandong University, Jinan, 250100, China
}

AND

LIN SUN

School of Mathematics

Shandong University, Jinan, 250100, China

Department of Mathematics

Changji University, Changji, 831100, China

\begin{abstract}
A total-k-coloring of a graph $G$ is a coloring of $V \cup E$ using $k$ colors such that no two adjacent or incident elements receive the same color. The total chromatic number $\chi^{\prime \prime}(G)$ of $G$ is the smallest integer $k$ such that $G$ has a total- $k$-coloring. Let $G$ be a graph embedded in a surface of Euler characteristic $\varepsilon \geq 0$. If $G$ contains no 3 -cycles adjacent to 4 -cycles, that is, no 3 -cycle has a common edge with a 4 -cycle, then $\chi^{\prime \prime}(G) \leq \max \{8, \Delta+1\}$.
\end{abstract}

Keywords: total coloring, embedded graph, cycle.

2010 Mathematics Subject Classification: 05C15.

\section{REFERENCES}

[1] M. Behzad, Graphs and Their Chromatic Numbers (Ph.D. Thesis, Michigan State University, 1965). 
[2] J.A. Bondy and U.S.R. Murty, Graph Theory with Applications (Macmillan Press Ltd., London, 1976).

[3] O.V. Borodin, On the total coloring of planar graphs, J. Reine Angew. Math. 394 (1989) 180-185.

[4] O.V. Borodin, Coupled colourings of graphs on a plane, Metody Diskret. Anal. 45 (1987) 21-27, in Russian.

[5] O.V. Borodin, A.V. Kostochka and D.R. Woodall, List edge and list total colourings of multigraphs, J. Combin. Theory Ser. B 71 (1997) 184-204. doi:10.1006/jctb.1997.1780

[6] O.V. Borodin A.V. Kostochka and D.R. Woodall, Total colorings of planar graphs with large maximum degree, J. Graph Theory 26 (1997) 53-59. doi:10.1002/(SICI)1097-0118(199709)26:1〈53::AID-JGT6〉3.0.CO;2-G

[7] G.J. Chang, J. Hou and N. Roussel, Local condition for planar graphs of maximum degree 7 to be 8-totally colorable, Discrete Appl. Math. 159 (2011) 760-768. doi:10.1016/j.dam.2011.01.001

[8] D. Du, L. Shen and Y. Wang, Planar graphs with maximum degree 8 and without adjacent triangles are 9-totally-colorable, Discrete Appl. Math. 157 (2009) 27782784 .

doi:10.1016/j.dam.2009.02.011

[9] T.R. Jensen and B. Toft, Graph Coloring Problems (Wiley Interscience, 1995).

[10] L. Kowalik, J.-S. Sereni and R. Škrekovski, Total-colorings of plane graphs with maximum degree nine, SIAM J. Discrete Math. 22 (2008) 1462-1479. doi: $10.1137 / 070688389$

[11] L. Shen and Y.Q. Wang, Total colorings of planar graphs with maximum degree at least 8, Sci. China Ser A: Math. 52 (2009) 1733-1742. doi:10.1007/s11425-008-0155-3

$[12]$ L. Shen and Y. Wang, On the 7 total colorability of planar graphs with maximum degree 6 and without 4-cycles, Graphs Combin. 25 (2009) 401-407. doi:10.1007/s00373-009-0843-y

[13] A.V. Kostochka, The total coloring of a multigraph with maximal degree 4, Discrete Math. 17 (1977) 161-163. doi:10.1016/0012-365X(77)90146-7

[14] A.V. Kostochka, An analogue of Shannon's estimate for complete colorings, Metody Diskret. Anal. 30 (1977) 13-22, in Russian.

[15] A.V. Kostochka, The total chromatic number of any multigraph with maximum degree five is at most seven, Discrete Math. 162 (1996) 199-214. doi:10.1016/0012-365X(95)00286-6

[16] B. Liu, J.F. Hou, J.L. Wu and G.Z. Liu, Total colorings and list total colorings of planar graphs without intersecting 4-cycles, Discrete Math. 309 (2009) 6035-6043. doi:10.1016/j.disc.2009.05.006 
[17] D.P. Sanders and Y. Zhao, On total 9-coloring planar graphs of maximum degree seven, J. Graph Theory 31 (1999) 67-73. doi:10.1002/(SICI)1097-0118(199905)31:1〈67::AID-JGT6〉3.0.CO;2-C

[18] V.G. Vizing, Some unsolved problems in graph theory, Uspekhi Mat. Nauk 23 (1968) 117-134, in Russian.

[19] B. Wang and J.-L. Wu, Total coloring of planar graphs with maximum degree seven, Inform. Process. Lett. 111 (2011) 1019-1021. doi:10.1016/j.ipl.2011.07.012

[20] P. Wang and J.-L. Wu, A note on total colorings of planar graphs without 4-cycles, Discuss. Math. Graph Theory 24 (2004) 125-135. doi:10.7151/dmgt.1219

[21] H.J. Wang, L.D. Wu, W.L. Wu, P.M. Pardalos and J.L. Wu, Minimum total coloring of planar graph, J. Global Optim. 60 (2014) 777-791. doi:10.1007/s10898-013-0138-y

[22] H.J. Wang, B. Liu, J.L. Wu and G.Z. Liu, Total coloring of embedded graphs with maximum degree at least seven, Theoret. Comput. Sci. 518 (2014) 1-9. doi:10.1016/j.tcs.2013.04.030

[23] H.J. Wang, B. Liu, J.L. Wu and B. Wang, Total coloring of graphs embedded in surfaces of nonnegative Euler characteristic, Sci. China Math. 57 (2014) 211-220. doi:10.1007/s11425-013-4576-2

[24] J.L. Wu and P. Wang, List-edge and list-total colorings of graphs embedded on hyperbolic surfaces, Discrete Math. 308 (2008) 6210-6215. doi:10.1016/j.disc.2007.11.044

[25] W.F. Wang, Total chromatic number of planar graphs with maximum degree ten, J. Graph Theory 54 (2007) 91-102. doi:10.1002/jgt.20195

Received 30 September 2016 Revised 30 March 2017 Accepted 30 March 2017 